\title{
TARGET INFLUENCE ON GROUND CONTROL POINTS (GCPS) IDENTIFICATION IN AERIAL IMAGES
}

\author{
Jonáš Hruška ${ }^{(I)}$, Luís Pádua ${ }^{(1,2)}$, Telmo Adão ${ }^{(1,2)}$, Emanuel Peres $^{(1,2)}$, José Martinho $^{(I)}$ and Joaquim J. Sousa $^{(1,2,)}$ \\ (1) University of Trás-os-Montes e Alto Douro, Portugal \\ (2) Centre for Robotics in Industry and Intelligent Systems, INESC-TEC, Portugal
}

\begin{abstract}
Unmanned aerial vehicles (UAVs) are used nowadays as a standard tool to derive very high-resolution geospatial data. However, UAV payload limitation imposes the use of not such reliable hardware affecting the georeferencing precision. In the literature it is possible to find numerous studies investigating the parameters influencing UAV-based products quality. Even if new photogrammetry methods could, in theory, avoid the use of ground control points (GCPs), they still play a key role to assure quality products. Nevertheless, usually only the number and distribution of GCPs are taking into account, since both change the geometric accuracy of the final products. In order to improve the understanding of the actual influence of GCPs, in this study we evaluate how can different physical characteristics affect GCPs identification in aerial images. The results demonstrate that GCPs' color, material, size and shape, among others, may influence a precise identification in aerial imagery.
\end{abstract}

Index Terms - UAV, Ground Control Points, GCPs,
Accuracy

\section{INTRODUCTION}

Nowadays, Unmanned Aerial Vehicles (UAVs) are used as a standard remote sensing platform to provide very high-resolution geospatial data. To deliver accurate geospatial UAV-based products for mapping applications it is crucial to assure the control of the most important parameters that may influence the final product quality. Due to UAV payload limitation, data acquisition is generally done by a light digital camera, not designed for photogrammetric purposes. Moreover, only small and not such reliable hardware (Global Navigation Satellite System - GNSS receiver and Inertial Measurement Unit - IMU) can be attached to UAVs, affecting the georeferencing precision, making it insufficient for certain types of projects.

To determinate the ground control coordinates the process called aerotriangulation is used. The aerotriangulation requires the transformation of image coordinates to ground coordinates using a set of points that must be clearly recognized in the aerial images. Those points are called Ground Control Points (GCPs) and play a crucial role in the whole process, effecting the final accuracy, which usually differs depending on the project goals. Rigorous GCPs are usually measured by traditional surveying methods like GNSS (Global Navigation Satellite System) receivers. This operation will increase the absolute accuracy of the UAV project, reducing the shift from meters to centimeters. However, in the preparation of a mission, usually only the number and the distribution of GCPs are taking into account, since both parameters change the geometric accuracy of the product. The GCPs should be spread homogeneously across the area, especially at the borders of the mapped area, otherwise the higher errors in accuracy can appear in these areas. The distribution and number of GCPs were tested in different studies [1-4].

Recommendations about good practices to place the targets are given by [5], in their image accuracy checkpoint collection guide. Other authors studied the influence of weather conditions in the target detection [6]. It was concluded that sunny days create clear light conditions increasing the contrast of the images and the GCPs placed on the ground could be hard to detect.

The number of GCPs can be reduced by adding two cross strips on each edge of the photogrammetric block, which will also strengthen the mathematic model within the bundle block adjustment computations [7].

GCPs still play a key role to assure quality products. For that reason, the main purpose of this study is to improve the understanding of the actual influence of GCPs' different parameters in the quality of the UAV-based products. To achieve this goal, the following related question is defined: How can different characteristics of GCPs affect the overall accuracy?

Regarding this topic - the use of GCPs - the only requirement generally found in the literature is that in order to assure reliability those points must be evenly distributed over the surveyed area. However, none of the previously referred studies focused on the patterns (shape and colors) or materials of the targets that compose the GCPs. In fact, from our knowledge, currently there are no studies that systematize the type of target to be used as GCP, depending on flight parameters and sensor type, but also on the target characteristics (color, material, size and shape, among others). For example, the flight altitude limits the detection of the mark on the image and, consequently, influences the accuracy of its identification. It is known that the more precisely the GCP is spotted in the images the higher positioning accuracy can be expected in final outputs [8].

Thus, the novelty of this research is to provide an improved understanding of the actual influence of different characteristics of GCPs, through a systematic analysis that considers the main influencing parameters: we hypothesized that not only the number of GCPs and distribution matter. To achieve the goals, three experiments were performed. In Experiment 1, optimal distribution and number of GCPs were tested in a vineyard area (few tens of hectares). In Experiment 2, the effect of missing GCPs was shown. Finally, in Experiment 3, the influence of targets parameters to georeferencing precision of the final output was evaluated using GCPs composed by different patterns, colors and materials. The experiments were focused on areas like dense forests, meadows and rural nature where no identifiable natural points related to the topographic features (building corners, water canals, traffic paintings on the roads) could be found and the man-made GCPs must be used. 


\section{MATERIALS AND METHODS}

The work was performed in the University of Trás-os-Montes e Alto Douro (UTAD) Campus (northern Portugal). The study site covers approximatively an area of 25 ha and includes a sport field area and a vineyard. This location was selected to perform the UAV flights and to develop the procedure because it is composed by two very distinct areas: the vineyard, with very few points that can be used as natural GCPs and the sportive area where is very easy to find natural targets, due to the regularity of some features. Two types of UAVs were used in this study: the eBee fixed-wing UAV, from senseFly (senseFly SA, Lausanne, Switzerland) and the Phantom 4 rotary-wing UAV, a quadcopter from DJI (DJ, Shenzhen, Guangdong, China). For each flight altitude, two flights were performed with eBee using the same flight plan, in order to collect data from different sensors: the Canon IXUS 127 HS camera, operating in the visible (RGB) region of the electromagnetic spectrum; and the Canon PowerShot ELPH 110 HS camera, operating in the red-edge (RE) region. RE is the spectral region where the plant's reflectance changes from low to high values $(715 \mathrm{~nm})$. GNSS measurements were taken by using the Mobile Mapper 100 receiver (Ashtech Infotech Pvt. Ltd., Mumbai, India) with an external antenna ASH 111661, capable of receiving the L1 and L2 frequencies of the GPS, G1 and G2 of GLONASS constellation, and L5 of the GALILEO constellation. The GNSS equipment was used in RTK mode from the Portuguese Network of Permanent Stations (ReNEP - Portuguese Network of Permanent Stations) and System of Virtual Reference GNSS Stations (SERVIR). In these conditions, the mean horizontal root mean square error (RMSE) was $1.9 \mathrm{~cm}$ and mean vertical RMSE was $2.6 \mathrm{~cm}$ from the points acquired during the survey, maximum and $\min$.

\section{RESULTS AND DISCUSSION}

\subsection{Experiment 1: Optimal distribution and number of GCPs}

The first experiment tested general suggestions from reviewed studies regarding the optimal number and distribution of GCPs. A total of 12 individual scenarios were set up (figure 1). The tests started with minimum number of 3 GCPs (theoretical minimum of GCPs for georeferencing the remote sensing data) till the last scenario where 14 GCPs were used. Possible combinations were tested with an intention to find out the best combination in terms of number and distribution allowing to achieve similar values in positioning accuracy.

The accuracy quality checking was carried out by using 6 checkpoints distributed in the area. For interpretation purposes the mean and RMSE values of checkpoints in the three coordinate directions are presented in figures 2 and 3 . The highest values of RMSE and mean are spotted in the first three scenarios. High error values are occurring especially in the z-coordinate at the 60 -meter altitude flight. Lower error values occur in scenarios with at least one GCP in the middle. The importance of GCP in the middle can be seen in the scenario 7, where the GCP is missing and the error of z-coordinate is big, especially in the highest altitude flights. The highest RMSE values were again spotted in the first three scenarios especially in z-coordinate of lower altitudes.

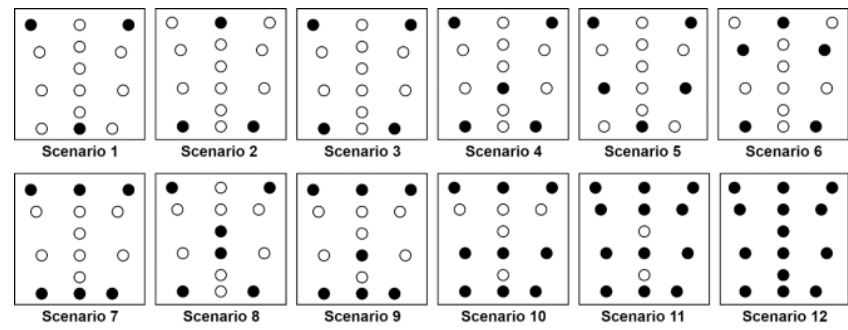

Figure 1 - The different configurations used in this study to test the influence of GCPs distribution and number

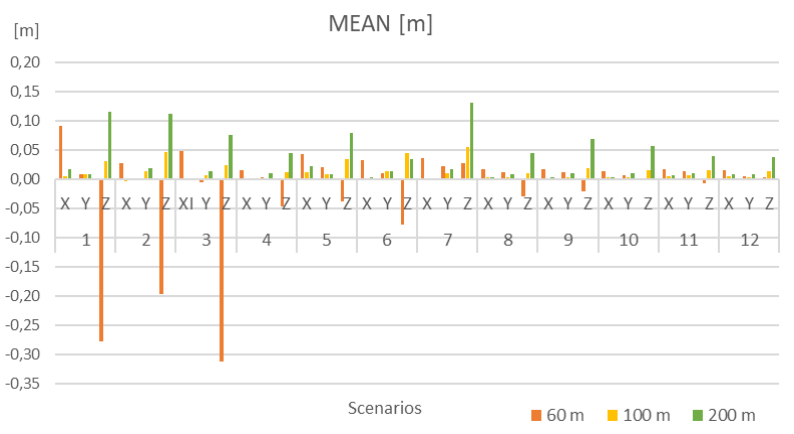

Figure 2 - MEAN values of checkpoints.

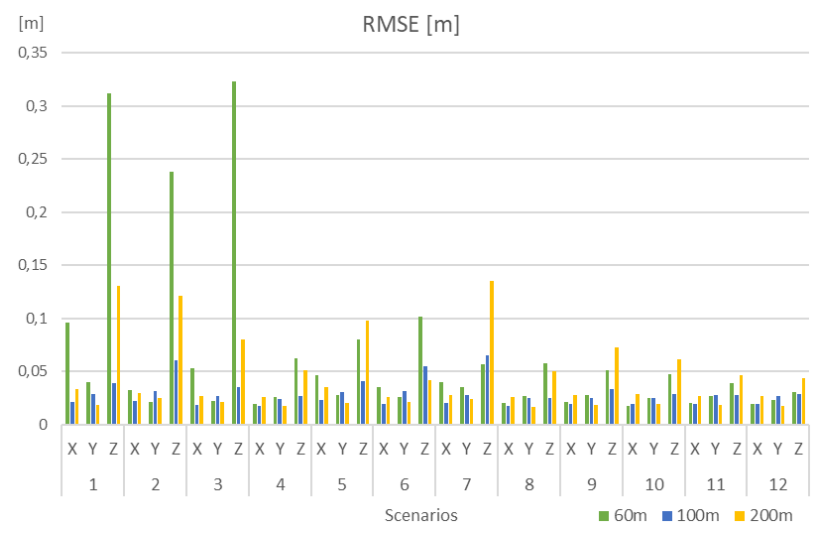

Figure 3 - RMSE values of checkpoints.

Scenario 4 achieved very similar values as the scenario 12 , with 14 GCPs, in $\mathrm{x}$ and $\mathrm{y}$-coordinates and that is why this set up was chosen as a good compromise to be used in Experiment 3.

\subsection{Experiment 2: The effect of missing GCPs}

The next experiment was carried out to assess the importance of having the GCPs spread all over the area. The experiment was performed using Phantom 4 at $60 \mathrm{~m}$ altitude with double grid flight plan. Two orthomosaics at each altitude were computed. In one case the model was georeferenced by using GCPs distributed in the whole surveyed area. In the other case the model was georeferenced by GCPs distributed only in the upper part of the area. The distribution of all GCPs can be seen in figure 4 . 


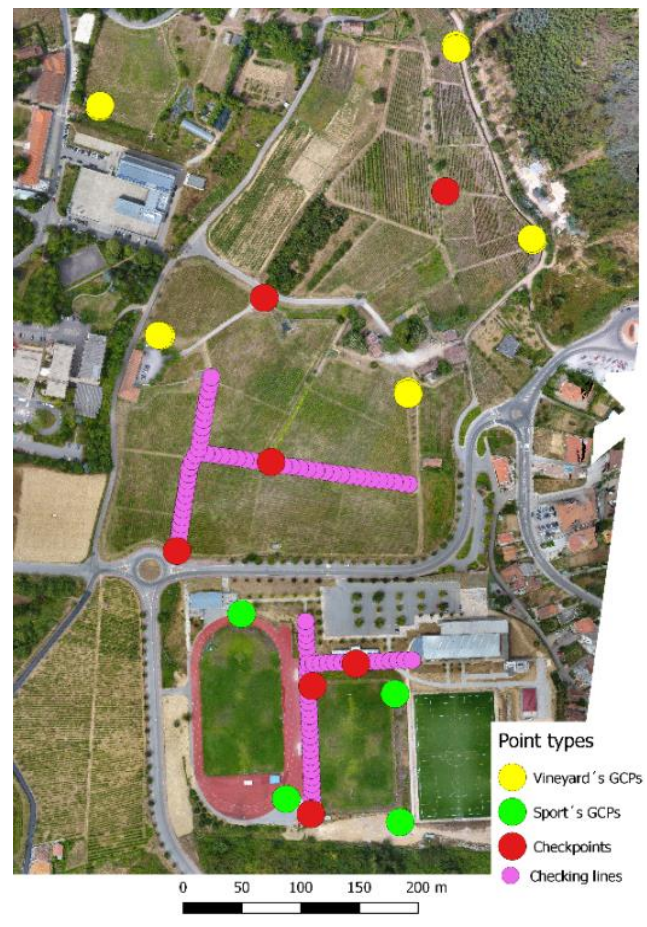

Figure 4 - GCPs distribution in vineyard and in sport areas.

For finding out of how the model might be influenced by the missing GCPs and in what direction the orthomosaic might be curved, the lines of points were collected in both horizontal and vertical directions with their positions measured by high precision GNSS receiver. The lines were collected in both parts of the area. The values from lines of points were then extracted from the computed models and compared with the actual values measured in the field. The z-coordinate values of lower vertical line shown that the closer to the area with GCPs the line is going, the smaller differences between the actual values and values in the model is spotted. The farthest point of the lower vertical line was almost 2 meters below the actual height getting to $90 \mathrm{~cm}$ difference of the closest point. In the horizontal direction of the line in lower part was spotted no tendency, but the heights from the model were about $80 \mathrm{~cm}$ below the actual heights across the whole line. In the upper lines the values in both models are very similar as expected because of the presence of GCPs.

\subsection{Experiment 3: Influence of targets parameters to georeferencing precision of the final output}

In the last experiment the different target parameters in terms of size, color and material were tested and answering whether the parameters could influence the accuracy of final models. Six types of targets were tested (table 1) grouped together into five distributed spots in the area (figure 5). GCP type 1 was made of red rubber material with yellow rectangle in the middle with black cross. Reflectance of this type of target was estimated as neutral. GCP type 2 was made of high reflective red rubber material with yellow cross and black rectangle in the middle. GCP types 3 and 4 were made based on Agisoft Photoscan recommended patterns, printed on two size white high reflective plastic material. GCP type 5 presented a yellow cross on black rounded low reflective plastic material. Finally, GCP type 6 was made of white plastic material with two black rectangles in the middle. The reflectivity of type 6 was evaluated as neutral.

Table 1 - Target types used in the experiment (Reflectance: " 0 " Neutral, “_" Low Reflectance, “+” High Reflectance.

\begin{tabular}{|c|c|c|c|c|}
\hline ID & Type & Size $[\mathrm{cm}]$ & Main colors & Reflectance \\
\hline 1 & & $93 \times 65$ & Red, yellow & 0 \\
\hline 2 & & $100 \times 90$ & Red, yellow & + \\
\hline 3 & & $30 \times 30$ & White, black & + \\
\hline 4 & & $100 \times 100$ & White, black & + \\
\hline 5 & & $23,5 \times \mathrm{r}$ & Black, yellow & - \\
\hline 6 & & $100 \times 100$ & White, black & 0 \\
\hline
\end{tabular}

Geo-referenced orthomosaics by each GCP type were compared at 4 altitudes $(50,100,200$ and $300 \mathrm{~m})$ by the RMSE value of checkpoints distributed in the area. The higher altitudes $(100,200$ and $300 \mathrm{~m})$ were flown by using senseFly eBee with Red-Edge and RGB cameras. The data of Red-Edge camera were tested only in the two highest altitudes. For orthomosaics of lower altitudes (50 and $100 \mathrm{~m}$ ) Phantom 4 with RGB camera was used. The same altitude of $100 \mathrm{~m}$ was taken by both UAVs to avoid any uncertainties because of the two different camera types. The results achieved by each GCP type can be seen in figure 5 and interpreted by RMSE values. In figure 5 , two groups for each type can be seen. In the first group are shown the RMSE values of $x$ and $y-$ coordinates, considered as two dimensional, planimetric positional error. In the second group are presented the values of $\mathrm{z}$ coordinates. The results of each type did not show a big difference range but still some conclusions could be stated. In the overall, the best results were achieved using GCP type 3, which is a small white target with PhotoScan pattern on it. Even though the pattern was not visible at all flying altitudes, the middle of the target was estimated by the using physical appearance of the target. The biggest target (type 4) where the pattern was not clearly identifiable the chance of estimating the middle of the target correctly was smaller as in case of type 3 . Interesting is to look at results of type 4 and type 6 . Smaller RMSEs of type 4 than type 6 were spotted. These two types were similar in terms of size and main color but differed in patterns. The view and the insight to pattern importance on GCPs from different altitudes in RGB imagery can be seen in figure 6 . It is noticeable that the pattern from lowest altitude $(50 \mathrm{~m})$ is visible on all GCPs, thus no issue at identifying the spot where the GNSS measurements were taken can occur. At $100 \mathrm{~m}$ altitude again all patterns were identifiable but the GCP type 3. Similar conditions appeared at $200 \mathrm{~m}$ altitude flight. At highest altitude $(300 \mathrm{~m})$ the only identifiable pattern was on GCP type 6 and slightly on GCP type 2 . 


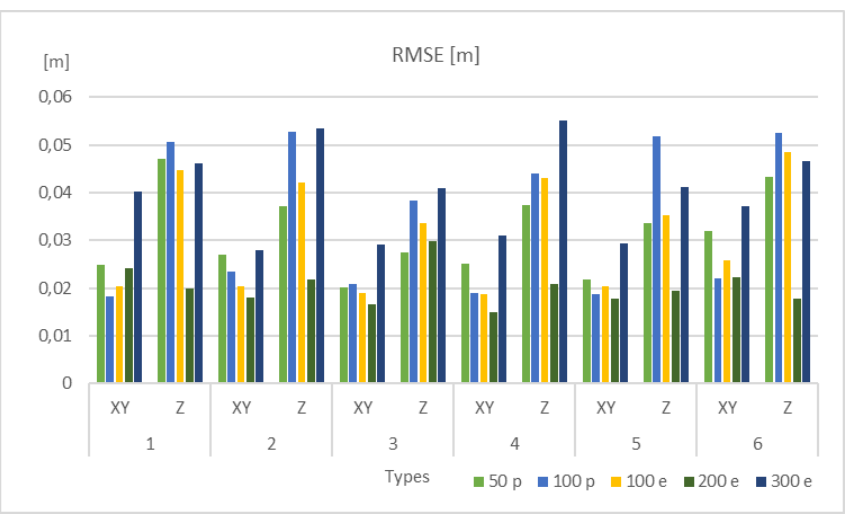

Figure 5 - RMSE values of checkpoints from mosaics calculated by GCP types (flying altitudes, $\mathrm{p}-$ Phantom $4, \mathrm{e}-\mathrm{eBee}$ ).

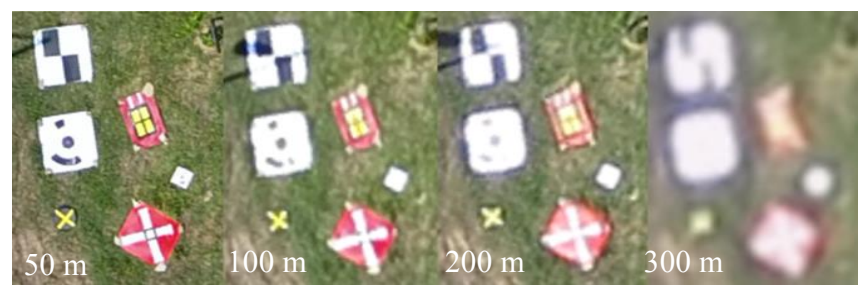

Figure 6 - The view at GCP area from different altitudes in RGB imagery.

In the Red-Edge imagery (figure 7) the color of GCP showed up its importance. The color revealed to be even more relevant parameter than in RGB imagery. It can be seen that the red color types (type 1 and 2) were hardly distinguishable from the red colored background characteristic for color infrared imagery. Hardly distinguishable was GCP type 5 as well. Its main black color blend with the background. On the other hand, white GCPs (type 3, 4 and 6) appeared expressive.

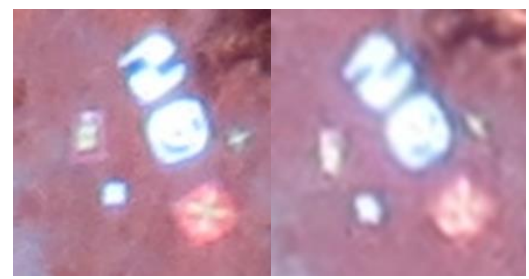

Figure 7 - The view at GCP area in RE imagery

\section{CONCLUSION}

The presented study helped in understanding the key role of using the proper GCP set up in the need of precise georeferenced outputs. In the first experiment the importance of having at least one GCP in the middle of the area was proven plus the fact that even smaller number of GCPs can achieve a similar accuracy like very dense spread GCPs. All set ups that had the GCP in the middle of the area provided lower RMSE values. Experiment 2 revealed how the final output can be twisted, when the GCPs are missing in certain part of the area. In our case the final model was twisted equally in the direction away from the area where the GCPs were used. In the last experiment it was found out that the pattern on GCP was not the key parameter for detecting it in images. The most relevant parameters appeared to be their size, color and material. In the case of size, we suggest using a target at least 6 times bigger than the GSD to preserve the physical appearance of the target in the images because of the resolution. The white color appeared as the most flexible color and was well detectable in both imagery sets. The importance of using non-reflective material was proven by experiencing the issues during marking the targets in processing software. In some images, the sun reflection made it hard to find the exact center of the target.

From the experiment came out the optimal target type. It is the simple pattern that was visible from all altitudes and is similar to GCP type 6 . But in the case of type 6 the gap between the cross of the tiles was spotted that's why we suggest using a small overlap of the tiles. Two diagonal lines will help in placing the GNSS receiver into the exact center (figure 8).

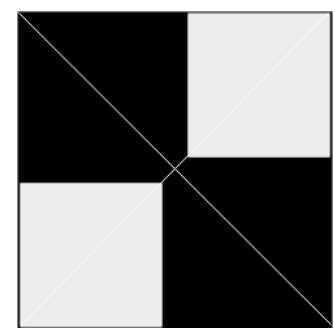

Figure 8 - The optimal target suggested from these experiments.

\section{REFERENCES}

[1] Agüera-Vega, F., Carvajal-Ramírez, F. and Martínez-Carricondo, P. (2017). Assessment of photogrammetric mapping accuracy based on variation ground control points number using unmanned aerial vehicle. Measurement, 98, 221-227.

[2] Barry, P. and Coakley, R. (2013). Field Accuracy Test of RPAS Photogrammetry. In ISPRS - International Archives of the Photogrammetry, Remote Sensing and Spatial Information Sciences (Vol. XL-1-W2, pp. 27-31).

[3] Ridolfi, E., Buffi, G., Venturi, S., \& Manciola, P. (2017). Accuracy Analysis of a Dam Model from Drone Surveys. Sensors, 17(8), 1777.

[4] Tahar, K. N. (2013). An evaluation on different number of ground control points in unmanned aerial vehicle photogrammetric block. ISPRS J Photogramm XL-2 (W2), 93-98.

[5] Sedorovich, A. and O'Hara, C. (2010). Retrieved from http://www.ee.co.za/wpcontent/uploads/legacy/PositionIT\%202009/PositionIT\%202010/image\%2 0accuracy.pdf

[6] Dandois, J. P., Olano, M. and Ellis, E. C. (2015). Optimal Altitude, Overlap, and Weather Conditions for Computer Vision UAV Estimates of Forest Structure. Remote Sensing, 7(10), 13895-13920.

[7] Lembicz, B. W. (2006). Minimizing Ground Control Points When GPS Photogrammetry Isn't Practical. ASPRS 2006 Annual Conference. Reno, Nevada. May 1-5, 2006. pp 1-13.

[8] Gonçalves, J.A. and Henriques, R. (2015). UAV photogrammetry for topographic monitoring of coastal areas. ISPRS Journal of Photogrammetry and Remote Sensing, 104, 101-111. 